Advances in Dynamical Systems and Applications (ADSA).

ISSN 0973-5321, Volume 15, Number 1, (2020) pp. 17-28

(C) Research India Publications

https://dx.doi.org/10.37622/ADSA/15.1.2020.17-28

\title{
Integrable Solutions for Caputo-Hadamard Implicit Fractional Order Differential Equations
}

\author{
Ahmed Zahed ${ }^{1}$, Samira Hamani ${ }^{1}$, and Johnny Henderson ${ }^{2}$ \\ ${ }^{1}$ Laboratoire des Mathematiques Appliqués et Pures, Université de Mostaganem, B.P. \\ 227, 27000, Mostaganem, Algerie. \\ ${ }^{2}$ Department of Mathematics, Baylor University, Waco, Texas 76798-7328, USA.
}

\begin{abstract}
In this paper we study the existence of integrable solutions for fractional order implicit differential equations with the Caputo-Hadamard fractional derivative of order $\alpha \in(1,2]$. Our results are based on Schauder's fixed point theorem and the Banach fixed point theorem.
\end{abstract}

Keywords: Boundary value problem, Existence, Caputo-Hadamard derivative , Implicit fractional differential equations, fixed point theorem.

AMS (MOS) Subject Classifications: 26A33, 34A08, 34A60, 34B15.

\section{INTRODUCTION}

In this paper we are concerned with the existence of integrable solutions of boundary values problem for the implicit fractional order differential equation,

$$
\begin{gathered}
{ }_{c}^{H} D^{\alpha} y(t)=f\left(t, y(t),{ }_{c}^{H} D^{\alpha} y(t)\right), \text { for a.e. } t \in J=[1, T], \quad 1<\alpha \leq 2, \\
y(1)=y_{1}, \quad y(T)=y_{T},
\end{gathered}
$$

where ${ }_{c}^{H} D^{\alpha}$ is the Caputo-Hadamard fractional derivative, and $f:[1, T] \times \mathbb{R} \times \mathbb{R} \rightarrow \mathbb{R}$ is a given function. Fractional order differential equations are generalizations of classical integer order differential equations. Fractional differential equations can 
describe many phenomena in various fields of applied sciences and engineering such as acoustics, control, signal processing, porous media, electrochemistry, viscoelasticity, rheology, polymer physics, proteins, electromagnetics, optics, medicine, economics, astrophysics, chemical engineering, chaotic dynamics, statistical physics, thermodynamics, biosciences, bioengineering, etc. See for example the monographs of Kilbas et al. [18], Podlubny [20], and the papers of Agarwal et al. [5], Momani et al. [19], Benhamida et al. [6, 7], Guerraiche et al. [14, 15], and the references therein.

Recently, considerable attention has been given to the existence of solutions of boundary value problems for implicit fractional differential equations. See for example the papers of Benchohra et al $[8,9,10]$ and references therein.

The Hadamard fractional derivative was introduced by Hadamard in 1892 [16]; this derivative differs from Caputo's derivative in two ways: the first is that its kernel contains a logarithmic function of arbitrary exponent, and the second is that the Hadamard derivative of a constant does not equal to 0 .

The Caputo-Hadamard fractional derivative given by Jarad et al. [13] is a modified Hadamard fractional derivative, but unlike the Hadamard fractional derivative, the Caputo-Hadamard fractional derivative of a constant is 0 , and this was inherited from the Caputo derivative. Recently, many researchers studied different fractional problems involving the Caputo Hadamrd derivative; see, for example, the papers of Shammack [21] and Y. Adjabi et al. [4].

To our knowledge, the literature on integrable solutions for fractional differential equations is very limited. El-Sayed and Hashem [11] studied the existence of integrable and continuous solutions for quadratic integral equations. El-Sayed and Abd El Salam considered $L^{p}$-solutions for a weighted Cauchy problem for differential equations involving the Riemann-Liouville fractional derivative.

In this paper we shall present two existence results for the problem (1)-(2). The first result relies on Schauder's fixed point theorem, while the second one is based up on the Banach contraction principle. Finally, we present an illustrative example.

\section{PRELIMINARIES}

In this section, we introduce notations, definitions, and preliminary facts that will be used in the remainder of this paper.

Let $C(J, \mathbb{R})$ be the Banach space of all continuous functions from $J$ into $\mathbb{R}$ with the supremum norm and let $L^{1}(J, \mathbb{R})$ be the Banach space of Lebesgue integrable functions 
$y: J \rightarrow \mathbb{R}$ with the norm

$$
\|y\|_{L^{1}}=\int_{J}|y(t)| d t
$$

Let us recall some definitions and properties of Hadamard fractional integration and differentiation. Let $\delta=t \frac{d}{d t}$, and set

$$
A C_{\delta}^{n}(J, \mathbb{R})=\left\{y: J \longrightarrow \mathbb{R}, \delta^{n-1} y(t) \in A C(J, \mathbb{R})\right\} .
$$

Definition 2.1. [18] The Hadamard fractional integral of order $r>0$ for a function $h \in L^{1}([1,+\infty), \mathbb{R})$ is defined as

$$
{ }^{H} I^{r} h(t)=\frac{1}{\Gamma(r)} \int_{1}^{t}\left(\log \frac{t}{s}\right)^{r-1} \frac{h(s)}{s} d s,
$$

provided the integral exists.

Example 2.2. Let $q>0$. Then

$$
{ }^{H} I_{1}^{q} \ln t=\frac{1}{\Gamma(2+q)}(\ln t)^{1+q} ; \text { for a.e. } t \in[1,+\infty) .
$$

Definition 2.3. [18] The Hadamard fractional derivative of order $r>0$ applied to the function $h \in A C_{\delta}^{n}([1,+\infty), \mathbb{R})$ is defined as

$$
\left({ }^{H} D_{1}^{q} h\right)(t)=\delta^{n}\left({ }^{H} I_{1}^{n-r} h\right)(t),
$$

where $n-1<r<n, n=[r]+1$, and $[r]$ is the integer part of $r$.

Definition 2.4. [13] For a given function $h \in A C_{\delta}^{n}([a, b], \mathbb{R})$, such that $0<a<b$, the Caputo-Hadamard fractional derivative of order $r>0$ is defined as follows:

$$
{ }_{c}^{H} D^{r} y(t)={ }^{H} D^{r}\left[y(s)-\sum_{k=0}^{n-1} \frac{\delta^{k} y(a)}{k !}\left(\log \frac{s}{a}\right)^{k}\right](t),
$$

where $\operatorname{Re}(\alpha) \geq 0$ and $n=[\operatorname{Re}(\alpha)]+1$.

Lemma 2.5. [13] Let $y \in A C_{\delta}^{n}([a, b], \mathbb{R})$ or $C_{\delta}^{n}([a, b], \mathbb{R})$ and $\alpha \in \mathbb{C}$. Then

$$
{ }^{H} I^{r}\left({ }^{H c} D^{r} y\right)(t)=y(t)-\sum_{k=0}^{n-1} \frac{\delta^{k} y(a)}{k !}\left(\log \frac{t}{a}\right)^{k} .
$$

Proposition 2.6. [21] Let $\alpha, \beta>0$ Then we have 
(1) $I^{\alpha}: L^{1}(J, \mathbb{R}) \rightarrow L^{1}(J, \mathbb{R})$, and if $f \in L^{1}(J, \mathbb{R})$, then

$$
I^{\alpha} I^{\beta} f(t)=I^{\beta} I^{\alpha} f(t)=I^{\alpha+\beta} f(t)
$$

(2) if $f \in L^{p}(J, \mathbb{R}), 1<p<\infty$, then $\left\|I^{\alpha} f(t)\right\|_{L^{p}} \leq \frac{(\log T)^{\alpha}}{\Gamma(\alpha+1)}\|f(t)\|_{L^{p}}$.

(3) The fractional order integral operator $I^{\alpha}$ is linear.

(4) The fractional order integral operator $I^{\alpha}$ maps $L^{1}(J, \mathbb{R})$ into itself.

(5) When $\alpha=n, I_{0}^{\alpha}$ is the $n$-fold integration.

Theorem 2.7. (Kolmogorov compactness criterion [21] ). Let $\Omega \subseteq L^{p}(J, \mathbb{R}), 1 \leq p \leq$ $+\infty$.. If

(i) $\Omega$ is bounded in $L^{p}(J, \mathbb{R})$ and

(i) $u_{n} \rightarrow u$ as $h \rightarrow 0$, uniformly with respect to $u \in \Omega$, then $\Omega$ is relatively compact in $L^{p}(J, \mathbb{R})$, where

$$
u_{h}(t)=\frac{1}{h} \int_{t}^{t+h} u(s) d s .
$$

Let us now recall a couple of fixed point theorems of which will be making use.

Theorem 2.8. [22](Schauder fixed point theorem) Let $E$ be a Banach space and $Q$ be a convex subset of $E$ and $T: Q \rightarrow Q$ be a compact and continuous map. Then $T$ has at least one fixed point in $Q$.

Theorem 2.9. [12](Banach fixed point theorem) Let $C$ be a non-empty closed subset of a Banach space $X$. Then any contraction mapping $T$ of $C$ into itself has a unique fixed point.

\section{MAIN RESULTS}

Let us start by defining what we mean by a solution of the problem (1)-(2).

Definition 3.1. A function $y \in L^{1}([1, T], \mathbb{R})$ is said to be a solution of (1)-(2) if there exists a function $x(t) \in L^{1}([1, T], \mathbb{R})$ with $x(t)=f(t, y(t), x(t))$ for a.e. $t \in[1, T]$ such that ${ }_{c}^{H} D^{\alpha} y(t)=x(t)$ and the function y satisfies conditions (2).

For the existence of solutions for the problem (1)-(2), we need the following auxiliary lemma. 
Lemma 3.2. For $1<\alpha \leq 2$, the solution of the BVP(1)-(2) can be expressed by the integral equation

$$
\begin{aligned}
y(t) & =\frac{1}{\Gamma(\alpha)} \int_{1}^{t}\left(\log \frac{t}{s}\right)^{\alpha-1} x(s) \frac{d s}{s}+y_{1} \\
& +\frac{y_{T}-y_{1}-\frac{1}{\Gamma(\alpha)} \int_{1}^{T}\left(\log \frac{T}{s}\right)^{\alpha-1} x(s) \frac{d s}{s}}{\log (T)} \log (t),
\end{aligned}
$$

where $x \in L^{1}([1, T], \mathbb{R})$ is the solution of the functional integral equation

$$
\begin{aligned}
x(t) & =f\left(t, \frac{1}{\Gamma(\alpha)} \int_{1}^{t}\left(\log \frac{t}{s}\right)^{\alpha-1} x(s) \frac{d s}{s}\right. \\
& \left.+y_{1}+\frac{y_{T}-y_{1}-\frac{1}{\Gamma(\alpha)} \int_{1}^{T}\left(\log \frac{T}{s}\right)^{\alpha-1} x(s) \frac{d s}{s}}{\log (T)} \log (t), x(t)\right),
\end{aligned}
$$

if and only if $y$ is a solution of the fractional boundary value problem

$$
\begin{gathered}
{ }_{c}^{H} D^{\alpha} y(t)=x(t) \text { for a.e. } t \in[1, T], \\
y(1)=y_{1}, \quad y(T)=y_{T} .
\end{gathered}
$$

Proof. Let ${ }_{c}^{H} D^{\alpha} y(t)=x(s)$ in equation (1). Then

$$
x(t)=f(t, y(t), x(t)) .
$$

Applying the Caputo-Hadamard fractional integral of order $r$ to both sides of (5), and by using Lemma 2.5 , we find that $y$ satisfies (6). Then Lemma 2.5 implies that

$$
y(t)=c_{1}+c_{2} \log (t)+{ }^{H} I^{\alpha} x(t) .
$$

The boundary conditions (6) imply that

$$
c_{1}=y_{1}
$$

and

$$
y(T)=\frac{1}{\Gamma(\alpha)} \int_{1}^{T}\left(\log \frac{T}{s}\right)^{\alpha-1} h(s) \frac{d s}{s}+y_{1}+c_{2}(\log T) .
$$

Hence

$$
c_{2}=\frac{y_{T}-y_{1}-\frac{1}{\Gamma(\alpha)} \int_{1}^{T}\left(\log \frac{T}{s}\right)^{\alpha-1} x(s) \frac{d s}{s}}{(\log T)} .
$$


Finally, we obtain the solution (3).

Conversely, it is clear that if $y$ satisfies equation (3), then equations (5)-(6) hold.

Let us introduce a list of assumptions.

(H1) $f: J \times \mathbb{R}^{2} \rightarrow \mathbb{R}$ is measurable in $t \in J$, for any $\left(u_{1}, u_{2}\right) \in \mathbb{R}^{2}$, and is continuous in $\left(u_{1}, u_{2}\right) \in \mathbb{R}^{2}$, for almost all $t \in J$.

(H2) There exist a positive function $a(t) \in L^{1}([1, T], \mathbb{R})$ and constants $b_{i}>0, i=1,2$, such that : $\left|f\left(t, u_{1}, u_{2}\right)\right| \leq a(t)+b_{1}\left|u_{1}\right|+b_{2}\left|u_{2}\right|$.

Our first result is based on the Schauder fixed point theorem

Theorem 3.3. Assume the assumptions (H1) and (H2) are satisfied. If

$$
\left(2 b_{1} \frac{(\log T)^{\alpha}}{\Gamma(\alpha+1)}+b_{2}\right)<1
$$

then the problem (1)-(2) has at least one solution on J.

Proof. Transform the problem (1)-(2 into a fixed point problem. Consider the operator

$$
H: L^{1}(J, \mathbb{R}) \rightarrow: L^{1}(J, \mathbb{R})
$$

defined by

$$
\begin{aligned}
(H x)(t) ; & f\left(t, \frac{1}{\Gamma(\alpha)} \int_{1}^{t}\left(\log \frac{t}{s}\right)^{\alpha-1} x(s) \frac{d s}{s}+y_{1}\right. \\
& \left.+\frac{y_{T}-y_{1}-\frac{1}{\Gamma(\alpha)} \int_{1}^{T}\left(\log \frac{T}{s}\right)^{\alpha-1} x(s) \frac{d s}{s}}{\log (T)} \log (t), x(t)\right) .
\end{aligned}
$$

The operator $H$ is well defined. Indeed, for each $x \in L^{1}([1, T], \mathbb{R})$, from assumptions (H1) and (H2), for

$$
r=\frac{\|a\|_{L^{1}}+2 b_{1}|T-1|\left(\left|y_{T}\right|+\left|y_{1}\right|\right)}{1-\left(2 b_{1} \frac{(\log T)^{\alpha}}{\Gamma(\alpha+1)}+b_{2}\right)},
$$


we have

$$
\begin{aligned}
\|H x\|_{L^{1}} & =\int_{1}^{T}|(H x)(t)| d t \\
& =\int_{1}^{T} \mid f\left(t, \frac{1}{\Gamma(\alpha)} \int_{1}^{t}\left(\log \frac{t}{s}\right)^{\alpha-1} x(s) \frac{d s}{s}\right. \\
& \left.+y_{1}+\frac{y_{T}-y_{1}-\frac{1}{\Gamma(\alpha)} \int_{1}^{T}\left(\log \frac{T}{s}\right)^{\alpha-1} x(s) \frac{d s}{s}}{\log (T)} \log (t), x(t)\right) \mid d t \\
& \leq \int_{1}^{T}|a(t)| d t \\
& +b_{1} \int_{1}^{T} \mid \frac{1}{\Gamma(\alpha)} \int_{1}^{t}\left(\log \frac{t}{s}\right)^{\alpha-1} x(s) \frac{d s}{s} \\
& +y_{1}+\frac{1}{\Gamma(\alpha)} \int_{1}^{T}\left(\log \frac{T}{s}\right)^{\alpha-1} x(s) \frac{d s}{s} \\
& +b_{2} \int_{1}^{T}|x(t)| d t \\
& \leq\|a\|_{L^{1}}+2 b_{1}|T-1|\left(\left|y_{T}\right|+\left|y_{1}\right|\right)+2 b_{1} \frac{(\log T)^{\alpha}}{\Gamma(\alpha+1)}\|x\|_{L^{1}}+b_{2}\|x\|_{L^{1}} \\
& \leq\|a\|_{L^{1}}+2 b_{1}|T-1|\left(\left|y_{T}\right|+\left|y_{1}\right|\right)+\left(2 b_{1} \frac{(\log T)^{\alpha}}{\Gamma(\alpha+1)}+b_{2}\right) r \\
\leq & r .
\end{aligned}
$$

Consider now the set, with $r$ as above,

$$
B_{r}=\left\{x \in L^{1}(J, \mathbb{R}):\|x\|_{L^{1}}<r\right\} .
$$

Clearly $B_{r}$ is nonempty, bounded, convex and closed. Now, we will show that $H B_{r} \subset B_{r}$. From (13) and (11), we get

$$
\|H x\|_{L^{1}} \leq\|a\|_{L^{1}}+2 b_{1}|T-1|\left(\left|y_{T}\right|+\left|y_{1}\right|\right)+2 b_{1} \frac{(\log T)^{\alpha}}{\Gamma(\alpha+1)}\|x\|_{L^{1}}+b_{2}\|x\|_{L^{1}} \leq r .
$$

Then $H B_{r} \subset B_{r}$. Assumption (H1) implies that $H$ is continuous.

Now, we will show that $H$ is compact; that is, $H B_{r}$ is relatively compact. Clearly $H B_{r}$ is bounded in $\left.L^{1}([1, T], \mathbb{R})\right)$; in particular, Condition (i) of the Kolmogorov compactness criterion is satisfied. It remains to show $(H x)_{h} \rightarrow(H x)$ as $h \rightarrow 0$ in $L^{1}([1, T], \mathbb{R})$ for each $x \in B_{r}$. 
Let $x \in B_{r}$, then we have

$$
\begin{aligned}
\left\|(H x)_{h}-(H x)\right\|_{L^{1}} & =\int_{1}^{T}\left|(H x)_{h}-(H x)\right| d t=\int_{1}^{T}\left|\frac{1}{h} \int_{t}^{t+h}(H x)(s) d s-(H x)(t)\right| d t \\
& \leq \int_{1}^{T}\left(\frac{1}{h} \int_{t}^{t+h}|(H x)(s)-(H x)(t)| d s\right) d t \\
& \leq \int_{1}^{T} \frac{1}{h} \int_{t}^{t+h} \mid f\left(s, \frac{1}{\Gamma(\alpha)} \int_{1}^{t}\left(\log \frac{t}{s}\right)^{\alpha-1} x(s) \frac{d s}{s}+y_{1}\right. \\
& \left.+\frac{y_{T}-y_{1}-\frac{1}{\Gamma(\alpha)} \int_{1}^{T}\left(\log \frac{T}{s}\right)^{\alpha-1} x(s) \frac{d s}{s}}{\log (T)} \log (t), x(s)\right) \\
& -f\left(t, \frac{1}{\Gamma(\alpha)} \int_{1}^{t}\left(\log \frac{t}{s}\right)^{\alpha-1} x(s) \frac{d s}{s}+y_{1}\right. \\
& \left.+\frac{y_{T}-y_{1}-\frac{1}{\Gamma(\alpha)} \int_{1}^{T}\left(\log \frac{T}{s}\right)^{\alpha-1} x(s) \frac{d s}{s}}{\log (T)} \log (t), x(t)\right) \mid d s d t .
\end{aligned}
$$

Since $x \in B_{r} \subset L^{1}([1, T], \mathbb{R})$ and assumption (H2) that implies $f \in L^{1}([1, T], \mathbb{R})$, we have

$$
\begin{gathered}
\frac{1}{h} \int_{t}^{t+h} \mid f\left(s, \frac{1}{\Gamma(\alpha)} \int_{1}^{t}\left(\log \frac{t}{s}\right)^{\alpha-1} x(s) \frac{d s}{s}\right. \\
\left.+y_{1} \frac{y_{T}-y_{1}-\frac{1}{\Gamma(\alpha)} \int_{1}^{T}\left(\log \frac{T}{s}\right)^{\alpha-1} x(s) \frac{d s}{s}}{\log (T)} \log (t), x(s)\right) \\
-f\left(t, \frac{1}{\Gamma(\alpha)} \int_{1}^{t}\left(\log \frac{t}{s}\right)^{\alpha-1} x(s) \frac{d s}{s}\right. \\
\left.+y_{1}+\frac{y_{T}-y_{1}-\frac{1}{\Gamma(\alpha)} \int_{1}^{T}\left(\log \frac{T}{s}\right)^{\alpha-1} x(s) \frac{d s}{s}}{\log (T)} \log (t), x(t)\right) \mid d s \rightarrow 0,
\end{gathered}
$$

as $h \rightarrow 0$.

\section{Hence}

$$
(H x)_{h} \rightarrow(H x) \text { uniformly as } h \rightarrow 0 .
$$

Then by the Kolmogorov compactness criterion, $H\left(B_{r}\right)$ is relatively compact. As a consequence of Schauder's fixed point theorem the BVP(1)-(2) has at least one solution in $B_{r}$. 
The following result is based on the Banach contraction principle

Theorem 3.4. Assume that the (HI) and the following condition hold.

(H3) There exist constants $k_{1}>0$ and $k_{2}>0$ such that,

$$
\left|f\left(t, x_{1}, y_{1}\right)-f\left(t, x_{2}, y_{2}\right)\right| \leq k_{1}\left|x_{1}-x_{2}\right|+k_{2}\left|y_{1}-y_{2}\right|, \quad x_{1}, x_{2}, y_{1}, y_{2} \in \mathbb{R}
$$

and

$$
\left(2 k_{1} \frac{(\log T)^{\alpha}}{\Gamma(\alpha+1)}+k_{2}\right)<1
$$

Then the problem (1)-(2) has a unique solution $y \in L^{1}(J, \mathbb{R})$.

Proof. We shall use the Banach contraction principle to prove that $H$, defined by (10), has a fixed point. Let $x, y \in L^{1}(J, \mathbb{R})$ and $t \in J$. Then we have,

$$
\begin{aligned}
|(H x)-(H y)| & =\mid f\left(t, \frac{1}{\Gamma(\alpha)} \int_{1}^{t}\left(\log \frac{t}{s}\right)^{\alpha-1} x(s) \frac{d s}{s}+y_{1}\right. \\
& \left.+\frac{y_{T}-y_{1}-\frac{1}{\Gamma(\alpha)} \int_{1}^{T}\left(\log \frac{T}{s}\right)^{\alpha-1} x(s) \frac{d s}{s}}{\log (T)} \log (t), x(t)\right) \\
& -f\left(t, \frac{1}{\Gamma(\alpha)} \int_{1}^{t}\left(\log \frac{t}{s}\right)^{\alpha-1} y(s) \frac{d s}{s}+y_{1}\right. \\
& \left.+\frac{y_{T}-y_{1}-\frac{1}{\Gamma(\alpha)} \int_{1}^{T}\left(\log \frac{T}{s}\right)^{\alpha-1} y(s) \frac{d s}{s}}{\log (T)} \log (t), y(t)\right) \mid \\
& \leq 2 k_{1} \frac{(\log T)^{\alpha}}{\Gamma(\alpha+1)} \int_{1}^{T}|x(s)-y(s)| d s+k_{2}|x(t)-y(t)| .
\end{aligned}
$$

Thus

$$
\begin{aligned}
\|(H x)-(H y)\|_{L^{1}} & \leq 2 k_{1} \frac{(\log T)^{\alpha}}{\Gamma(\alpha+1)}\|x-y\|_{L^{1}}+k_{2}\|x-y\|_{L^{1}} \\
& \leq\left(2 k_{1} \frac{(\log T)^{\alpha}}{\Gamma(\alpha+1)}+k_{2}\right)\|x-y\|_{L^{1}} .
\end{aligned}
$$

Consequently by (13), $H$ is a contraction. As a consequence of the Banach contraction principle, we deduce that $H$ has a unique fixed point which is a solution of the problem (1)-(2). 


\section{AN EXAMPLE}

Consider the boundary value problem,

$$
\begin{gathered}
{ }_{c}^{H} D^{\frac{3}{2}} y(t)=\frac{1}{4 e^{t+5}\left(1+|y(t)|+\left.\right|_{c} ^{H} D^{\frac{3}{2}} y(t) \mid\right)}, \text { for a.e. } t \in J=[1, e], \quad 1<\alpha \leq 2, \\
y(1)=0, y(e)=1 .
\end{gathered}
$$

Set

$$
f(t, u, v)=\frac{1}{4 e^{t+5}(1+|u|+|v|)}, t \in J \times[0, \infty) \times[0, \infty) .
$$

Let $u_{1}, u_{2}, v_{1}, v_{2} 1 \mathbb{R}$ and $t \in J$. Then we have

$$
\begin{aligned}
\left|f\left(t, u_{1}, v_{1}\right)-f\left(t, u_{2}, v_{2}\right)\right| & =\left|\frac{1}{4 e^{t+5}}\left(\frac{1}{\left(1+u_{1}+v_{1}\right)}-\frac{1}{\left(1+u_{2}+v_{2}\right)}\right)\right| \\
& \leq\left|\frac{1}{4 e^{t+5}}\left(\frac{\left|u_{1}-u_{2}\right|+\left|v_{1}-v_{2}\right|}{\left(1+u_{1}+v_{1}\right)\left(1+u_{2}+v_{2}\right)}\right)\right| \\
& \leq \frac{1}{4 e^{t+5}}\left(\left|u_{1}-u_{2}\right|+\left|v_{1}-v_{2}\right|\right) \\
& \leq \frac{1}{4 e^{5}}\left|u_{1}-u_{2}\right|+\frac{1}{4 e^{5}}\left|v_{1}-v_{2}\right| .
\end{aligned}
$$

Hence the condition (H3) holds with $k_{1}=k_{2}=\frac{1}{4 e^{5}}$. We shall check that condition (13) is satisfied with $T=e$. Indeed

$$
2 k_{1} \frac{(\log T)^{\alpha}}{\Gamma(\alpha+1)}+k_{1}=\frac{1}{2 e^{5} \Gamma(\alpha+1)}+\frac{1}{4 e^{5}}<1 .
$$

Then by Theorem 3.4 the problem (14)-(15) has a unique integrable solution on $[1, e]$.

\section{REFERENCES}

[1] S. Abbas, M. Benchohra, J.R. Graef and J. Henderson, Implicit Fractional Differential and Integral Equations: Existence and Stability, De Gruyter, Berlin,2018.

[2] S. Abbas, M. Benchohra and G.M. N'Guerekata, Topics in Fractional Differential Equations, Springer, New York, 2012

[3] S. Abbas, M. Benchohra and G.M. N'Guerekata, Advanced Fractional Differential and Integral Equations, Nova Science Publishers, New York, 2015 
[4] Y.Adjabi, F.Jarad, D.Baleanu and T.Abdeljawad, On Cauchy problems with Caputo Hadamard Fractional Derivatives, J. Comput. Anal. Appl.21 (4)(2016), 661-681.

[5] R.P. Agarwal, M. Benchohra and S. Hamani, A survey on existence results for boundary value problems for nonlinear fractional differential equations and inclusions,Acta Applicandae Math. 109, No. 3 (2010), 973-1033.

[6] W. Benhamida, S. Hamani and J. Henderson, Boundary value problems for Caputo-Hadamard fractional differential equations, Advances in the Theory of Nonlinear Analysis and its Applications. 2 (2018) No. 3, 138-145.

[7] W. Benhamida and S. Hamani, Measure of noncompactness and Caputo-Hadamard fractional differential equations in Banach spaces, Eurasian Bulletin of Mathematics. EBM, 1 (2018), NO 3, 98-106.

[8] M. Benchohra and M. S. Souid, Integrable solutions for implicit fractional order differential equations. Transylvanian Journal of Mathematics and Mechanics 6 (2014), 2, 101-107.

[9] M. Benchohra and M. S. Souid, $L^{1}$-solutions for implicit fractional order differential equations with nonlocal condition, Filomat 30, No 6 (2016), 1485-1492.

[10] M. Benchohra and M. S. Souid, Integrable solutions for Implicit fractional order functional differential equations with infinite delay, Archivum Mathematicum (BRNO) 51 (2015), 67-76 .

[11] A.M.A. El-Sayed and H.H.G. Hashem, Integrable and continuous solutions of a nonlinear quadratic integral equation, Electron.J. Qual. Theory Differ. Equ. No 25, 2008, 1-10.

[12] A. Granas and J. Dugundji, Fixed Point Theory. Springer-Verlag, New York, 2003.

[13] F. Jarad, T. Abdeljawad and D. Beleanu, Caputo-type modification of the Hadamard fractional derivative, Advances in Difference Equations. 2012, 142, (2012).

[14] N. Guerraiche, S. Hamani and J. Henderson, Initial value problems for fractional functional differential inclusions with Hadamard type derivative, Archivum Mathematicum. 52 (2016), 263 - 273. 
[15] N. Guerraiche, S. Hamani and J. Henderson, Boundary value problems for differential inclusions with integral and anti-periodic conditions, Communications on Applied Nonlinear Analysis. 23 (2016), No. 3, 33 - 46.

[16] J. Hadamard, Essai sur l'etude des fonctions donnees par leur development de Taylor, J. Mat. Pure Appl. Ser. 8 (1892), 101-186.

[17] R. Hilfer, Applications of Fractional Calculus in Physics, World Scientific, Singapore, 2000.

[18] A. A. Kilbas, H. M. Srivastava and J. J. Trujillo, Theory and Applications of Fractional Differential Equations. North-Holland Mathematics Studies, 204, Elsevier Science B.V., Amsterdam, 2006.

[19] S. M. Momani, S. B. Hadid and Z. M. Alawenh, Some analytical properties of solutions of diifferential equations of noninteger order, Int. J. Math. Math. Sci. 2004 (2004), 697-701.

[20] I. Podlubny, Fractional Differential Equations, Academic Press, San Diego, 1999.

[21] W. Shammakh, A study of Caputo-Hadamard-Type fractional differential equations with nonlocal boundary conditions, Journal of Function Spaces, 2016.

[22] K. Deimling, Nonlinear Functional Analysis, Springer-Verlag, 1985. 